\title{
Evolution of Candida Diagnostics: Are We There Yet?
}

\section{Natesan $\mathrm{SK}^{*}$}

John D. Dingell VA Medical Center and Wayne State University, USA

*Corresponding author: Suganthini Krishnan Natesan, Chief, Division of Infectious Diseases, John D. Dingell VA Medical Center, Associate Professor of Medicine, Wayne State University Detroit, MI USA, Tel: 313-576-3057; Fax: 313-576-1242; Email:

\section{Review Article}

Volume 2 Issue 1

Received Date: May 29, 2019

Published Date: June 19, 2019

DOI: 10.23880 /oajmms-16000104

Suganthini.krishnannatesan@va.gov

\section{Abstract}

Invasive candidiasis (IC) is an important cause of nosocomial blood stream infections and deep tissue infections, not only in immunocompromised patients but also in immunocompetent patients admitted to intensive care units. Despite the advent of novel azoles and echinocandins over the last few years, IC continues to be associated with an unacceptable mortality rate of $\sim 30 \%$. The critical factors that will have a favorable impact on morbidity and mortality of IC, include early diagnosis and timely initiation of appropriate antifungal therapy. Blood culture is currently the gold standard for diagnosis of candidemia. Currently available blood culture systems detect a dismal $50 \%$ of IC and therefore unreliable. Hence it is imperative that non -culture based diagnostic tests are developed, validated and standardized to enhance the diagnostic sensitivity of invasive candidiasis. The last three decades have witnessed the intermittent flow of several diagnostic tests, most of which failed to stand the test of time as they lacked sensitivity and specificity. This article provides an overview of various non -culture methods that have been unsuccessfully tried in the past, details the currently available advanced novel diagnostics and helps understand their strengths and limitations. It also provides a guide for physicians to interpret test results from a clinical perspective that will translate into judicious use of antifungal agents. Such a strategic approach will enhance patient care and decrease mortality associated with IC.

Keywords: Candidemia; Invasive Candidiasis; Mortality; Diagnosis; Echinocandins

Abbreviations: IC: Invasive candidiasis; NNISS: National Nosocomial Infections Surveillance System; MALDI-TOF: Matrix Associated Laser Desorption Ionization-Time of Flight response.

\section{Introduction}

Invasive candidiasis (IC) is an important cause of nosocomial blood stream infections and deep tissue infections. Candida species are considered normal commensals of the gastrointestinal and genitourinary tracts and are known to cause serious infections in immunocompromised patients and patients admitted to intensive care units $[1,2]$. The extent of infection depends on local and systemic immune responses in the host and ranges from superficial infections involving skin and mucosa to deep infections with invasion of blood stream and other organs. Factors that increase the risk of IC include prolonged stay in intensive care unit, abdominal surgery, broad spectrum antibiotic therapy, total parenteral nutrition, central venous lines, burns, 


\section{Open Access Journal of Mycology \& Mycological Sciences}

hematopoietic stem cell transplant, solid organ transplantation and use of immunosuppressive agents [1].

The National Nosocomial Infections Surveillance System (NNISS) reports Candida species as the $4^{\text {th }}$ most common cause of nosocomial bloodstream infections. Despite the advent of the novel azoles and echinocandins, IC continues to be associated with an unacceptably high mortality rate of $\sim 30 \%$. Blood culture is currently the gold standard for diagnosis of candidemia. Given the fact that most advanced blood culture systems for candida detect a dismal $50 \%$ of infections, they cannot be relied upon as sole diagnostic tools [3,4]. Poor sensitivity of blood culture systems and delays in diagnosis and initiation of antifungal therapy contribute to the high incidence of mortality with IC. Clearly there has been an unmet challenge and need for improving candida diagnostics.

Several non-culture diagnostic tests serve as adjuncts to blood cultures to facilitate rapid diagnosis of IC [5]. The beta-D-glucan assay measures the antigenic components of Candida cell wall and has been widely used over the last several years in North America [6]. The recent introduction of two highly sensitive tests namely the MALDI-TOF (Matrix Associated Laser Desorption Ionization-Time of Flight response) [7] and the T2 Candida nano-diagnostic panel, seem to have revolutionized the diagnosis of IC and taken it to a different dimension [8]. The following review provides a brief overview of the evolution of candida diagnostics, difficulties in interpreting test results, their strengths and limitations, and guide clinicians interpret test results from a clinical perspective [9].

\section{Available Non-Culture Diagnostic Tests For IA}

\section{North America}

1. 1, 3, -beta-D-glucan

2. MALDI-TOF

3. T2 panel (exclusively for candidemia)

4. Verigene

5. Candida PCR (not FDA approved)

\section{Globally (Not FDA Approved In The USA)}

1. Mannan antigen test

2. Anti-mannan IgG test

3. C. albicans germ tube antibody test (CAGTA)

History of Evolution of Biological, Metabolic and Enzyme Markers in Candida Diagnostics

1. Serum D-Arabinitol

\section{Enolase immunoassay}

3. Serum aspartyl proteinase in blood and urine

4. Immunoblot detection of candida antigen in blood and urine

5. Cell wall mannoprotein antigen (CWMP)

\section{Background}

Blood culture is the gold standard for the diagnosis of candidemia. However, it is known to have poor sensitivity and potentially misses candidemia in $\sim 30-40 \%$ of cases. Candida diagnosis in late 1980s started with the introduction of a lysis centrifugation isolator blood culture system. This system involved lysis of leucocytes and release of candida from blood cells. The resulting sediment was plated on 5 different agar plates and incubated for growth. Although crude, this system greatly improved detection of candida from blood stream infection. In high-risk patients this system detected $\sim 73 \%$ of candidemia with a mean time to detection of candida growth of $\sim 2.2$ days [10]. However, detection was not consistent, with wide variations noted between studies (25-82\%) in patients suspected of IC.

Earlier literature suggested that candida ligand interaction with host receptors contributed to clearance of candida from bloodstream by entrapment in the sinusoidal endothelium of the liver. Based on this observation, several cellular enzymes, metabolites and cell wall antigens have been evaluated as potential markers for candidemia [11].

\section{Metabolites of Candida}

\section{Serum D-Arabinitol}

One of the first in the series of tests was the detection of arabinitol, a metabolite from candida. Several methods were implemented to differentiate host arabinitol from candida arabinitol and to adjust for renal dysfunction [12]. The major hurdles were the requirement of a gas liquid chromatography test that was labor intensive, and issues with cross reactivity with serum D-mannitol rendering the test obsolete.

\section{Enolase}

Enolase is an enzyme (antigen) of the glycolytic pathway of Candida albicans. It was thought to form immune-complexes with host immunoglobulins during pathogenesis. It was shown that there was immunological cross-reactivity between enolase and a 48kDa antigen in serum of patients with IC. It was detected in serum using 


\section{Open Access Journal of Mycology \& Mycological Sciences}

a liposomal rhodamine sandwich immunoassay with a sensitivity in histologically proven cases of about $52 \%$ [13]. However, it wasn't clear if it could detect enolases from non-albicans candida, and multiple blood samples were needed for detection. Also, as there was no in vitro process to disassociate enolase from immune complexes, there was a concern if immune complex formation would drastically decrease the sensitivity of the test and hence it was unsuccessful.

\section{Aspartyl Proteinase (AP)}

Candida albicans secretes an aspartyl proteinase during its growth in proteinaceous substrates. Several extracellular proteinases have been shown to be involved in penetration, severe vasoconstriction, disseminated intravascular coagulation, deactivation of immunoglobulins, and escape from phagolysosomes contributing to virulence in C. albicans and C. tropicalis. In an in vitro rabbit model, an enzyme immunoassay was able to detect AP in urine within 24 hours of intravenous infection. It was able to differentiate candida colonization from deep infection and was positive only for $C$. albicans and negative for Aspergillus, Cryptococcus and other Candida species [14]. Although the test appeared promising, it's very narrow spectrum of detection and lack of evaluation in humans led to its failure.

\section{Antigenic Markers}

\section{Cell Wall Mannoproteins (CWMP)}

Despite ongoing efforts, research in this field staggered, with a combination of tests that were used for diagnosing invasive candidiasis. Improvisation of markers and techniques led to evaluation of cell wall components (CWMP) of candida as antigenic markers of infection. Surface carbohydrates called CWMP are antigenic components present in cell wall of Candida species that are secreted during IC. They circulate as CWMP-antibody immune complexes and need to be disassociated for detection using enzyme immunoassay, radioimmunoassay or reverse passive latex agglutination (RPLA). There are 2 different epitopes identified namely $A$ and $B$ of which epitope $A$ has been identified in majority if cases using specific monoclonal antibodies. The sensitivity of this test was found to be less predictable, it occurred in $31-90 \%$ of cases of IC and its detection depended on frequency of sampling, level of host immunity, type of assay used and was only specific for $C$. albicans and C. tropicalis [15]. This test was commercialized for a while but issues with poor sensitivity and variability with different tests led to its eventual failure.
All the above non-culture antigen and metabolite tests were gradually developed based on research that spanned more than a decade. However, none of these tests were sensitive and specific enough to be accepted as reliable markers of IC. They served as tests supplemental to standard blood culture for the diagnosis of IC [16].

\section{Candida DNA Amplification Using Polymerase Chain Reaction (PCR)}

Studies on PCR were delayed due to the presumption (i) that candida was rapidly cleared from the blood and escape detection by PCR and (ii) that certain inhibitors in blood could inhibit the PCR. The first target selected for amplification by PCR was the target of azoles namely the single copy of the target gene sequence that encodes cyctochrome P-450 14-alpha-lanosterol demethylase Earlier studies that amplified this gene by PCR consisted of lysing leucocytes and erythrocytes in blood using detergents, lysing candida cell walls using zymolase, digesting proteins with proteinase, digesting human and candida DNA with DNAse, extracting DNA, purifying DNA and performing PCR using agarose gel electrophoresis. The detection limit using this procedure was $120 \mathrm{CFU} / \mathrm{ml}$. As fewer than $100 \mathrm{CFU} / \mathrm{ml}$ of organisms were expected in blood of patients with candidemia, the sensitivity of this test was deemed to be poor. Since then several studies have been published using different targets with modified, validated, fine-tuned versions of this test.

One of the upgraded versions of DNA amplification introduced in 1997 was a PCR assay for rapid identification of Candida species from positive blood culture bottles. It used fungal-specific universal primers for DNA amplification and species-specific probes that detected 5 different species of clinical significance namely C. albicans, C. tropicalis, C. parapsilosis, C. glabrata and C. krusei amplicons. The detection limit of this test when combined with an EIA was as few as 100 cells per $200 \mu \mathrm{l}$. Recently, with global emergence of multi-drug resistant $C$. auris, amplicons to detect these species have added to the platform [17-20]. This was considered well within the sensitivity that was needed to detect $100 \%$ of all positive blood cultures [1]. As noted, this test detected Candida from positive blood culture bottles and not from whole blood. It is important to recognize that enzymatic inhibitors are needed when whole blood is used because of relatively lower concentration of candida in whole blood and a higher concentration of PCR inhibitors.

\section{Current Scenario}

The mannan, anti-mannan antibody and the C. albicans germ tube antibody (CAGTA) tests are widely employed in 
the European countries and not FDA approved in North America. The sensitivity of these tests was extremely poor and ranged from 25 to $50 \%$ and varied between candidemia and deep-seated candidiasis [6].

\section{Beta D Glucan (BDG)}

A cell wall constituent used as a marker for candida infections and currently used is BDG. It is present in Candida and Aspergillus species and is notably absent in Mucorales and Cryptococcus [7]. Most commercialized tests are indirect calorimetric or turbid-metric assays of serum that measure and quantify BDG mediated activation of a horseshoe crab coagulation cascade. False positive tests have been reported with exposure to beta lactam antibiotics, Candida or Aspergillus colonization, receipt of human blood products, hemodialysis filters, enteric nutrition, disruption of GI tract integrity and with cellulose dressings. The negative predictive value of this test is more than $85 \%$ with sensitivity and specificity of $\sim 65 \%$ and $\sim 75 \%$ respectively. Therefore, a negative test result with questionable clinical risk factors could justify discontinuation of antifungal therapy. Wide availability of this test has allowed physicians to use empiric antifungal agents judiciously, thereby supporting antifungal stewardship activities in hospitals $[6,18]$. In addition to the false positives outlined above, another limitation of this test is the use of batch testing once or twice weekly to collect multiple samples and perform the test on a single use 96-well plates. This could lead to potential delays in turn-around time and affect patient care.

\section{Advanced PCR Tests}

Several modified versions of PCR tests are currently available. A meta-analysis of 54 studies involving 5000 patients reported that whole blood sample had a better sensitivity than serum, with a sensitivity and specificity of $\sim 95 \%$ for candidemia and $\sim 75 \%$ for other deep-seated infections. The detection limit of this test is $\sim 1-10$ $\mathrm{CFU} / \mathrm{ml}$. Better sensitivity was also noted when panfungal rRNA or cyp450 genes were employed as targets and when candida specific assays were used instead of broad range multiplex assays [21-23].

\section{Matrix Assisted Laser Desorption Ionization- Time of Flight Mass Spectrometry (MALDI-TOF)}

Recently, (MALDI-TOF MS) has emerged as an effective tool for identification and diagnosis of candida infections. The major limitation of the technology is that diagnosis is possible only if the laboratory database contains peptide mass fingerprints of the specific Candida species (Figure 1). However, as most clinical strains of candida are represented in the database, it is not an issue for candida infections. The test is currently used in several institutions as it is rapid, sensitive, and economical in terms of both labor and costs involved $[7,24,25]$.

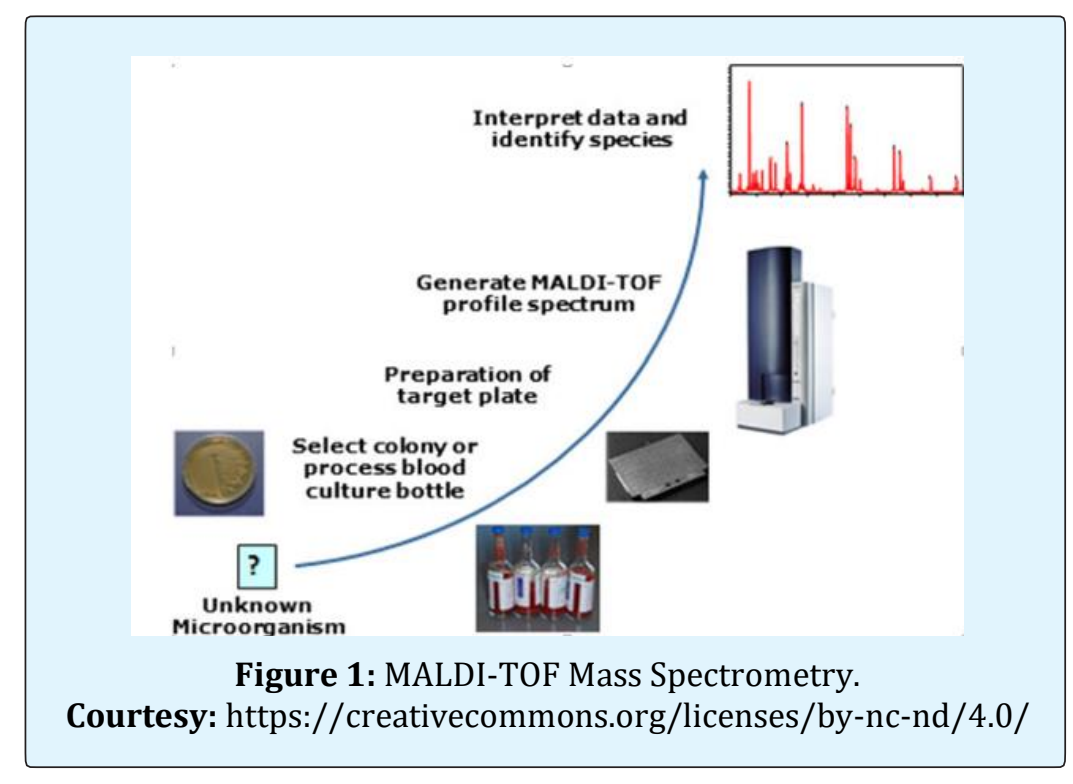

Given its high sensitivity and specificity, it seems to be slowly replacing PCR tests. It is also being evaluated for its potential role in taxonomy, strain typing and to identify antifungal drug-resistance. Costs of acquiring the equipment, and complexities involved in software training and analysis continue to pose major restrictions that curtails its widespread use. 


\section{T2 Candida Panel Using Magnetic Resonance}

The recent development and introduction of a novel qualitative diagnostic platform called "the T2 candida panel" seems to offer a breakthrough in the diagnostics of candidemia $[8,26,27]$. It is the first of its kind and is based on cutting edge technology that involves PCR based amplification and magnetic resonance. It is the only FDA- approved fungal pathogen detection system that detects candida directly from whole blood without awaiting results of a positive blood culture (Figure 2). All other FDA approved blood tests for candida detection rely on a positive blood culture, which is known to miss about $50 \%$ of candidemia.

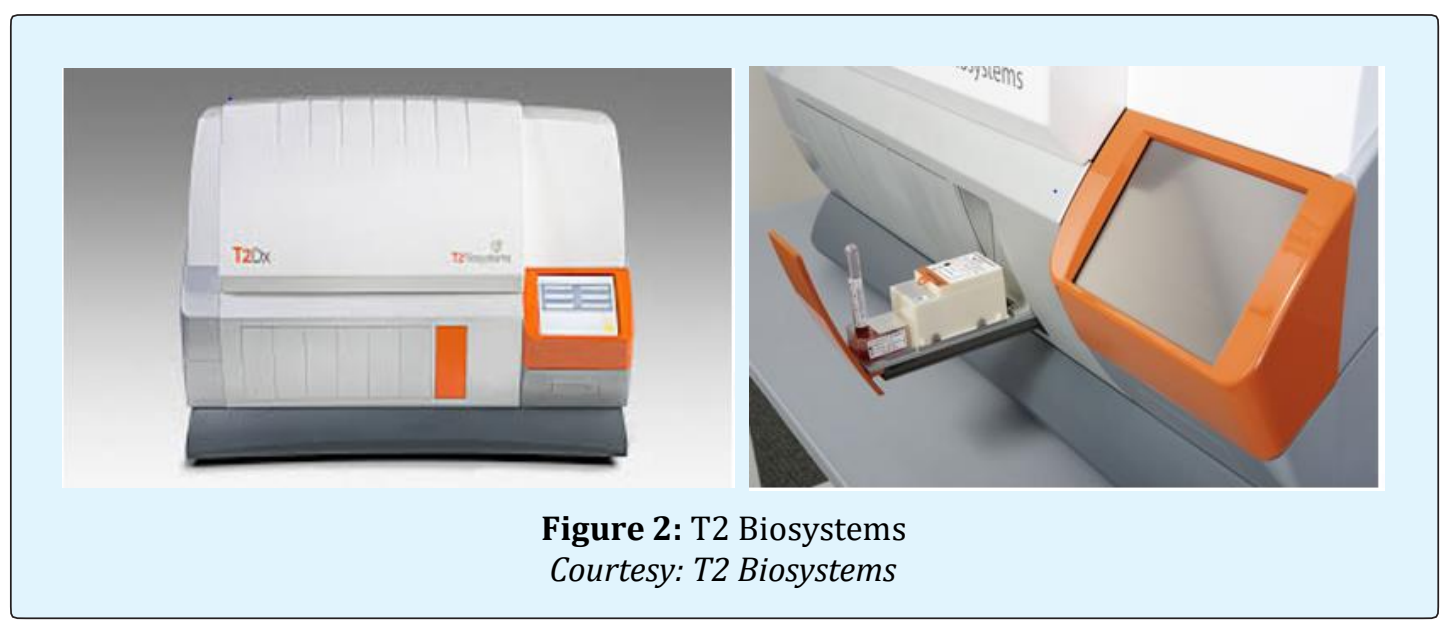

FDA approval of this test was based on data obtained from a multi-center DIRECT trial that tested $>1500$ control patients with candida negative blood cultures, 6 patients with candida positive blood cultures and 250 blood samples to which known concentration of candida (1-100 CFU/ml) was added.

The T2 candida panel also identified 37 cases of candidemia in patients who were receiving antifungal therapy that were missed by standard blood cultures drawn simultaneously, in a multi-center DIRECT2 study. Available evidence suggests that this test is superior to blood cultures, with a mean time to species identification of 3-5 hours, and consistently demonstrates a high sensitivity of $90 \%$ and specificity of $\sim 98-99 \%$. The T2 panel detects 5 different candida species from K2EDTA whole blood samples and is performed on a completely automated T2Dx instrument. The 5 different Candida species of clinical significance namely $C$. albicans, $C$. tropicalis, C. parapsilosis, C. glabrata and C. krusei. This test offers a unique platform that entails a 2-step process. The first step comprises of basic molecular diagnostics that involves DNA amplification using gene specific primers. The second step encompasses detection of the amplified product by amplicon-induced agglomeration of super-magnetic particles that are measured using T2 magnetic resonance (T2MR). The detection limit is 1-3 $\mathrm{CFU} / \mathrm{ml}$ which is several-fold higher than that reported by PCR assay mentioned above [26,27].
The few limitations of this test are as follows: (a) indeterminate results with thawed blood samples (b) limited to candidemia and not for deep tissue infections (c) significance of culture negative and $\mathrm{T} 2$ positive cases (d) impact of antifungal therapy $€$ interpretation and prognosis of serial test results. Despite its high sensitivity, it is still considered an adjunct to standard microbiological blood cultures.

\section{Discussion/A Clinician Perspective}

As detailed above, the field of candida diagnostics has gradually evolved over several decades. In addition to tests described above, the tenacious efforts included a voluminous array of tests that were essentially unsuccessful. It included traditional crude germ tube assays [28], monoclonal antibody [29] and GLABRATA tests [30], latex agglutination [31] and immunosorbent assays [32], internally transcribed spacer 2 region [33], mass spectrometry [34] and pan fungal amplicon assays [35], dual path platforms [36], DNA amplification [37], interleukin 17A and kynurenine [38] and anti-phosphopeptidomannan antibody assays [39], ultimately leading to the current T2 assay [40]. The current standard for candida testing includes BDG, PCR and T2 panel as adjuncts to standard blood culture test. Most data available so far on these tests pertain to candidemia and not for deep seated infections. In fact, the sensitivity and specificity of these tests for deep tissue infections drops down to the range of $30-90 \%$. Given the high sensitivity of 


\section{Open Access Journal of Mycology \& Mycological Sciences}

PCR and T2 candida panel of $>90 \%$, false positive test results are expected. It is important for clinicians to acknowledge that the positive and negative predictive values of a test for candidemia are dependent on the pretest probability of candidemia. Therefore, these tests are recommended only in situations where the clinical scenario raises the concern for candidemia based on evaluation of host risk factors and a clinical presentation. Given the high negative predictive value of the test, clinicians must be willing to consider stopping antifungal treatment in low risk patients especially if blood cultures, PCR and/or T2 panel are reported negative. Highly sensitive tests must be interpreted with caution as false positives have been reported in $\sim 20-30 \%$ cases using T2 panel. It is also reported that $\sim 25 \%$ of clinicians were not willing to stop antifungal therapy despite negative T2 test results. Nevertheless, T2 tests were ordered (when available) in all patients along with blood cultures. In high risk patients, where the the clinician has a strong index of suspicion for candidemia (based on host risk factors and clinical criteria), and the pre-test probability of the test is high, patients will be continued on empiric antifungal therapy regardless of non-culture test results. In such situations it would be prudent to withhold PCR or T2 panel tests. In low risk patients, there is always a concern for false positive test results and hence positive test result needs to be interpreted from a clinical perspective. These tests serve as best adjuncts to standard blood cultures in patients who are at moderate risk for IC.

\section{Conclusion and Clinical Perspective}

Highly sensitive non-culture tests such as PCR and T2 Candida panel have been long overdue tests in the field of candida diagnostics. They seem to be revolutionizing the diagnosis of candidemia especially cases missed by standard blood cultures [41]. Their sensitivity clearly supersedes blood cultures, and false positives are expected given their high sensitivity. A fundamental understanding of these test results and their interpretation is crucial for clinicians managing IC.

Prior to introducing these tests in their laboratory, hospitals need to acknowledge the implications of test results from stewardship and reimbursement perspective [42]. The major concern would be over-reporting of falsepositive candidemia as central venous line-related blood stream infection (CLABSI) that may have reimbursement implications for the hospital. Hence, formulation of a protocol that determines the best scenarios for using this test to benefit patients and aid clinicians is essential. It needs to be implemented in coordination with the laboratory, infection control, stewardship and infectious disease teams prior to introducing this test in an institution. A stratified approach, would streamline and provide a guide for rational use of these tests. Judicious utilization of these tests will help in diagnosis of candidemia missed by blood cultures, avoid overreporting of candidemia, avoid inappropriate use of antifungal agents and improve overall patient care. On the other hand, inadvertent and inappropriate use of these tests will lead to more confusion, misinterpretation of results, overuse of antifungal agents and adversely affect patient care $[43,44]$. The field of candida diagnostics has witnessed tremendous progress over decades, from unrefined, crude tests introduced in 1970s to the currently available technologically advanced sophisticated tests $[45,46]$. It is vital that these highly sensitive novel diagnostic tests are interpreted from a clinical standpoint, especially when there is discrepancy between culture and non-culture test results. Clinicians need to understand the pre-test probability of IC to avoid spurious diagnosis, use these tests wisely and learn to interpret test results from a clinical perspective. Such a strategic approach would definitely facilitate early diagnosis and treatment of IC and decrease mortality in these patients.

\section{References}

1 Bassetti M, Garnacho-Montero J, Calandra T, Kullberg B, Dimopoulos G, et al. (2017) Intensive care medicine research agenda on ninvasive fungal infection in critically ill patients. Intensive Care Med 43(9): 1225-1238.

2 McCarthy MW, Walsh TJ (2018) Candidemia in the cancer patient: diagnosis, treatment, and future directions. Expert Rev Anti Infect Ther 16(11): 849854.

3 Pappas PG, Lionakis MS, Arendrup MC, OstroskyZeichner L, Kullberg BJ (2018) Invasive candidiasis. Nat Rev Dis Primers 4: 18026.

4 Spampinato C, Leonardi D (2013) Candida infections, causes, targets, and resistance mechanisms: traditional and alternative antifungal agents. Biomed Res Int 2013: 204237.

5 Yeo SF, Wong B (2002) Current status of nonculture methods for diagnosis of invasive fungal infections. Clin Microbiol Rev 15(3): 465-484.

6 Kedzierska A, Kochan P, Pietrzyk A, Kedzierska J (2007) Current status of fungal cell wall components in the immunodiagnostics of invasive fungal 


\section{Open Access Journal of Mycology \& Mycological Sciences}

infections in humans: galactomannan, mannan and (1-->3)-beta-D-glucan antigens. Eur J Clin Microbiol Infect Dis 26(11): 755-766.

7 Bao JR, Master RN, Azad KN, Schwab DA, Clark RB, et al. (2018) Rapid, Accurate Identification of Candida auris by Using a Novel Matrix-Assisted Laser Desorption Ionization-Time of Flight Mass Spectrometry (MALDI-TOF MS) Database (Library). J Clin Microbiol 56(4).

8 Clancy CJ, Nguyen MH (2018) T2 magnetic resonance for the diagnosis of bloodstream infections: charting a path forward. J Antimicrob Chemother 73(4): iv2-iv5.

9 Clancy CJ, Nguyen MH (2018) Non-Culture Diagnostics for Invasive Candidiasis: Promise and Unintended Consequences. J Fungi (Basel) 4(1).

10 Kirkley BA, Easley KA, Basille BA, Washington JA (1993) Controlled clinical comparison of two lysisbased blood culture systems, isolator and Septi Chek Release, for detection of bloodstream infections. J Clin Microbiol 31(8): 2114-2117.

11 SiewFah Yeo, Brian Wong (2002) Current Status of Nonculture Methods for Diagnosis of Invasive Fungal Infections. Clinical Microbiology Reviews 15(3): 465484.

12 Stradomska TJ, Bobula-Milewska B, Bauer A, Mielniczuk Z, Dabkowska M, et al. (2005) Urinary Darabinitol/L-arabinitol levels in infants undergoing long-term antibiotic therapy. J Clin Microbiol 43(10): 5351-5354.

13 Reiss E, Morrison CJ (1993) Nonculture methods for diagnosis of disseminated candidiasis. Clin Microbiol Rev 6(4): 311-323.

14 Naglik JR, Challacombe SJ, Hube B (2003) Candida albicans secreted aspartyl proteinases in virulence and pathogenesis. Microbiol Mol Biol Rev 67(3): 400428.

15 Martinez JP, Gil ML, Lopez-Ribot JL, Chaffin WL (1998) Serologic response to cell wall mannoproteins and proteins of Candida albicans. Clin Microbiol Rev 11(1): 121-141.

16 Nawrot U, Kowalska-Krochmal B, Sulik-Tyszka B, Kozak M, Swietek K, et al. (2015) Evaluation of blood culture media for the detection of fungi. Eur J Clin Microbiol Infect Dis 34(1): 161-167.
17 Wellinghausen N, Siegel D, Winter J, Gebert S (2009) Rapid diagnosis of candidaemia by real-time PCR detection of Candida DNA in blood samples. J Med Microbiol 58(Pt 8): 1106-1111.

18 McKeating C, White PL, Posso R, Palmer M, Johnson E, et al. (2018) Diagnostic accuracy of fungal PCR and $\beta$ d-glucan for detection of candidaemia: a preliminary evaluation. J Clin Pathol 71(5): 420-424.

19 Safavieh M, Coarsey C, Esiobu N, Memic A, Vyas JM, et al. (2017) Advances in Candida detection platforms for clinical and point-of-care applications. Crit Rev Biotechnol 37(4): 441-458.

20 Arastehfar A, Fang W, Badali H, Vaezi A, Jiang W, et al. (2018) Low-Cost Tetraplex PCR for the Global Spreading Multi-Drug Resistant Fungus, Candida auris and Its Phylogenetic Relatives. Front Microbiol 9: 1119 .

21 Ao W, Klonoski J, Berlinghoff E, Jensen J, Afroz T, et al. (2017) Rapid Detection and Differentiation of Clinically Relevant Candida Species Simultaneously from Blood Culture by Use of a Novel Signal Amplification Approach. J Clin Microbiol 56(1).

22 Elges S, Arnold R, Liesenfeld O, Kofla G, Mikolajewska A, et al. (2017) Prospective evaluation of the SeptiFAST multiplex real-time PCR assay for surveillance and diagnosis of infections in haematological patients after allogeneic stem cell transplantation compared to routine microbiological assays and an in-house real-time PCR method. Mycoses 60(12): 781-788.

23 Sidiq F, Hoostal M, Rogers SO (2016) Rapid identification of fungi in culture-negative clinical blood and respiratory samples by DNA sequence analyses. BMC Res Notes 9: 293.

24 Pulcrano G, Iula DV, Vollaro A, Tucci A, Cerullo M, et al. (2013) Rapid and reliable MALDI-TOF mass spectrometry identification of Candida non-albicans isolates from bloodstream infections. J Microbiol Methods 94(3): 262-266.

25 Girard V, Mailler S, Chetry M, Vidal C, Durand G, et al. (2016) Identification and typing of the emerging pathogen Candida auris by matrix-assisted laser desorption ionisation time of flight mass spectrometry. Mycoses 59(8): 535-538.

26 Mylonakis E, Clancy CJ, Ostrosky-Zeichner L, Garey KW, Alangaden GJ, et al. (2015) T2 magnetic 
resonance assay for the rapid diagnosis of candidemia in whole blood: a clinical trial. Clin Infect Dis 60(6): 892-899.

27 Mylonakis E, Zacharioudakis IM, Clancy CJ, Nguyen MH, Pappas PG (2018) Efficacy of T2 Magnetic Resonance Assay in Monitoring Candidemia after Initiation of Antifungal Therapy: The Serial Therapeutic and Antifungal Monitoring Protocol (STAMP) Trial. J Clin Microbiol 56(4).

28 Crist AE Jr, Dietz TJ, Kampschroer K (1996) Comparison of the MUREX C. albicans, Albicans-Sure, and BactiCard Candida test kits with the germ tube test for presumptive identification of Candida albicans. J Clin Microbiol 34(10): 2616-2618.

29 Marcilla A, Monteagudo C, Mormeneo S, Sentandreu R (1999) Monoclonal antibody 3H8: a useful tool in the diagnosis of candidiasis. Microbiology 145: 695-701.

30 Freydiere AM, Robert R, Ploton C, Marot-Leblond A, Monerau F, et al. (2003) Rapid identification of Candida glabrata with a new commercial test, GLABRATA RTT. J Clin Microbiol 41(8): 3861-3863.

31 Marot-Leblond A, Beucher B, David S, Nail-Billaud S, Robert R (2006) Development and evaluation of a rapid latex agglutination test using a monoclonal antibody to identify Candida dubliniensis colonies. J Clin Microbiol 44(1): 138-142.

32 Prince HE, Yeh C, Alem N, Asalkhou M, Hamedi N, et al. (2008) Evaluation of enzyme-linked immunosorbent assays for detecting circulating antibodies to Candida albicans. J Clin Lab Anal 22(4): 234-238.

33 Landlinger C, Baskova L, Preuner S, Willinger B, Buchta V, et al. (2009) Identification of fungal species by fragment length analysis of the internally transcribed spacer 2 region. Eur J Clin Microbiol Infect Dis 28(6): 613-622.

34 Zehm S, Schweinitz S, Würzner R, Colvin HP, Rieder J (2012) Detection of Candida albicans by mass spectrometric fingerprinting. Curr Microbiol 64(3): 271-275.

35 Mandviwala T, Shinde R, Kalra A, Sobel JD, Akins RA (2010) High-throughput identification and quantification of Candida species using high resolution derivative melt analysis of panfungal amplicons. J Mol Diagn 12(1): 91-101.
36 Gunasekera M, Narine M, Ashton M, Esfandiari J (2015) Development of a Dual Path Platform (DPP®) immunoassay for rapid detection of Candida albicans in human whole blood and serum. J Immunol Methods 424: 7-13.

37 Baskova L, Landlinger C, Preuner S, Lion T (2007) The Pan-AC assay: a single-reaction real-time PCR test for quantitative detection of a broad range of Aspergillus and Candida species. J Med Microbiol 56(9): 11671173.

38 Krause R, Zollner-Schwetz I, Salzer HJ, Valentin T, Rabensteiner J, et al. (2015) Elevated levels of interleukin $17 \mathrm{~A}$ and kynurenine in candidemic patients, compared with levels in noncandidemic patients in the intensive care unit and those in healthy controls. J Infect Dis 211(3): 445-451.

39 Mattsby-Baltzer I, Pinel C, Yugueros Marcos J, Kondori N, Potton L, et al. (2015) IgG1 anti-cell wall and IgG2 anti-phosphopeptidomannan antibodies in the diagnosis of invasive candidiasis and heavy Candida colonization. Med Mycol 53(7): 725-735.

40 Pfaller MA, Wolk DM, Lowery TJ (2016) T2MR and T2Candida: novel technology for the rapid diagnosis of candidemia and invasive candidiasis. Future Microbiol 11(1): 103-117.

41 Beyda ND, Amadio J, Rodriguez JR, Malinowski K, Garey KW, et al. (2018) In Vitro Evaluation of BacT/Alert FA Blood Culture Bottles and T2Candida Assay for Detection of Candida in the Presence of Antifungals. J Clin Microbiol 56(8).

42 Hamdy RF, Zaoutis TE, Seo SK (2017) Antifungal stewardship considerations for adults and pediatrics. Virulence 8(6): 658-672.

43 Bilir SP, Ferrufino CP, Pfaller MA, Munakata J (2015) The economic impact of rapid Candida species identification by T2Candida among high-risk patients. Future Microbiol 10(7): 1133-1144.

44 Posch W, Heimdorfer D, Wilflingseder D, Lass-Florl C (2017) Invasive candidiasis: future directions in nonculture based diagnosis. Expert Rev Anti Infect Ther 15(9): 829-838.

45 Ruangritchankul K, Chindamporn A, Worasilchai N, Poumsuk U, Keelawat S, et al. (2015) Invasive fungal disease in university hospital: a PCR-based study of autopsy cases. Int J Clin Exp Pathol 8(11): 1484014852. 


\section{Open Access Journal of Mycology \& Mycological Sciences}

46 Gutierrez J, Maroto C, Piedrola G, Martin E, Perez JA (1993) Circulating Candida antigens and antibodies: useful markers of candidemia. J Clin Microbiol 31(9): 2550-2552. 